\title{
Unilateral Basement Membrane Zone Alteration of the Regenerated Laminar Region in Equine Chronic Laminitis
}

\author{
Atsutoshi KUWANO ${ }^{1)}$, Takanori UENO ${ }^{1)}$, Yoshinari KATAYAMA ${ }^{2)}$, Toshio NISHIYAMA ${ }^{3)}$ and Katsuhiko ARAI ${ }^{3)}$ \\ ${ }^{1)}$ Clinical Science \& Pathobiology Division, Equine Research Institute, Japan Racing Association, 321-4 Tokami-cho, Utsunomiya-shi, \\ Tochigi 320-0856, 2) Epizootic Research Station (Tochigi-branch), Equine Research Institute, Japan Racing Association, 1400-4 Shiba, \\ Kokubunji-machi, Shimotsuga-gun, Tochigi 329-0412 and ${ }^{3}$ Department of Tissue Physiology, Tokyo University of Agriculture and \\ Technology, Fuchu, Tokyo 183-8509, Japan
}

(Received 18 June 2004/Accepted 29 March 2005)

\begin{abstract}
Between the laminar epidermis and the laminar dermis of laminar region (LR) in equine foot, it can be observed the basement membrane zone (BMZ), which is composed of a basement membrane and its accompaniments like the hemidesmosome and anchoring fibril. Alteration in the BMZ in equine laminitis is possibly related with not only development but also recovery outcome and recurrence of this disease. However, there is little known about the structure of the BMZ during the recovery phase of this disease. To assess the condition of the BMZ of LR affected by chronic laminitis, the tissue was examined in three cases at two weeks, four weeks and three months after the onset of laminitis, using pathological, immunohistochemical and electron microscopic techniques. Histologically in all laminitis cases, there was a regenerated laminar epidermis with proliferating keratinocytes between the Stratum medium and the dermis, but it included the undeveloped secondary epidermal laminae (ud-SELs) structure in one side of the primary epidermal laminae, especially in the part of the deep area of LR. Immunohistochemical results were positive for the anti-type IV collagen, anti-type VII collagen and anti-laminin 5 antibodies in the most BMZs. However, partial BMZs adjacent to the ud-SELs were negative for the anti-type VII collagen and anti-laminin 5 antibodies. Ultrastructurally, in the BMZ of the ud-SEL, the lamina densa and the lamina lucida were present. In contrast, the anchoring fibrils and the hemidesmosomes were either absent, or present at lower than normal levels. In conclusion, the present study indicated that the part of regenerated LR in chronic laminitis was not able to fully restore to construct the BMZ for a long time, especially in the unilateral side of laminar epidermis. It might be related with recurrence of this disease. KEY WORDS: basement membrane zone, chronic laminitis, collagen, equine, laminin 5 .
\end{abstract}

J. Vet. Med. Sci. 67(7): 685-691, 2005

The integumental tissues of the foot are important in supporting the horse's entire body weight during static and dynamic loading conditions. If severe intractable hoof disease occurs, the horse may lose not only the ability to run, but also to stand. Laminitis is one such intractable hoof condition and has a recurrent nature. Many horses have suffered from this disease over extended periods, resulting in significant treatment costs, and therefore the equine industry has a great deal of interest in clarifying the pathogenesis and developing treatment methods $[5,9,12]$.

Anatomically, the laminar region in foot displays a complex 3-dimensional structure consisting of the primary epidermal lamina (PEL) and the secondary epidermal lamina (SEL) which interdigitate with their dermal counterparts [24]. These dermal and epidermal components are separated by the basement membrane zone (BMZ), which is composed of a basement membrane and its accompaniments like the hemidesmosome and anchoring fibril [23]. The hemidesmosome, anchoring fibril and other collagenous ultramicroscopic structures in the BMZ unite the integument and the distal phalanx to form a single functional entity that suspends the appendicular skeleton within the hoof capsule. This suspensory apparatus of the distal phalanx is responsible for achieving painless force transfer between the

\footnotetext{
* Present address: Kuwano, A., Clinical Science \& Pathobiology Division, Equine Research Institute, Japan Racing Association, 321-4 Tokami-cho, Utsunomiya-shi, Tochigi 320-0856, Japan.
}

ground and the skeleton during loading. The primary pathological feature associated with laminitis is degradation of the integumental tissues of the LR [25]. This degradation results in partial or total separation of the laminar dermis (LD) and laminar epidermis (LE) [26]. In systemic acute laminitis disruption to the dermo-epidermal junction of the integument is known to be caused by the BMZ degradation [26]. This degradation results from the up-regulation of specific zinc-dependent endopeptidases such as matrix metalloproteinases 1, 2 and 9 (MMPs 1, 2 and 9) [14, 22, 27]. Its pathogenic process is similar to that of human epidermolysis bullosa $[17,19,31]$. In the case of grafted human skin, further more, insufficient formation of $\mathrm{BMZ}$ proteins including type IV collagen, type VII collagen and laminin 5 cause detachment of the epidermis from the dermis [7, 10]. Though such deterioration can be observed within the dermo-epidermal junction in early phase of acute laminitis, dissection of the chronically affected hoof shows existence of the hypertrophic LE between the ectopic white line tissue (lamellar wedge) and the distal phalanx [16, 18, 29]. This suggests that regeneration of the dermo-epidermal junction occurs due to active proliferation of the keratinocytes during the recovery phase. Nevertheless, few studies have been conducted on this pathologic features of the BMZ during the healing process. Thus, it is not clear whether the regenerated LE would have a normal BMZ structure. This information is important for determining how to treat chronically 
affected hooves, and for predicting whether a laminitic hoof would be prone to repeated traumatic tearing at the dermoepidermal junction. In the present study, major components of the BMZ were examined in cases of chronic laminitis using pathological, immunohistochemical and electron microscopic techniques to clarify this important aspect of laminitis pathology.

\section{MATERIALS AND METHODS}

Horses: Three thoroughbred racehorses (Case 1: a two year-old filly, Case 2: a three year-old female, Case 3: a seven year-old male), which were diagnosed with chronic laminitis based on typical gait and radiological evidence of a submural radiolucent air line and palmar rotation of the distal phalanx (DP rotation) [21], were studied. Duration of condition in Cases 1, 2 and 3 had been for two weeks, four weeks and three months, respectively. Severe DP rotation was observed radiographically in all laminitis cases. The cause of laminitis was not specified in each case. A control racehorse without evidence of hoof damage (three-year-old female), which was euthanized because of catastrophic fracture of the front third metacarpal bone, was used for comparative microscopic and electron microscopic analyses.

Dissection: Dissection followed the modified method of the previously reported one [18]. From the midline dead center (MDC) of the hoof capsule [28], two parasagittal cuts were made $10 \mathrm{~mm}$ medial and lateral respectively of the plane of the MDC using an electric band saw (Makita Co., Ltd., Aichi, Japan). The resultant sagittal was divided along the plane of the MDC to give two parasagittal sample blocks (A and B). The medial parasagittal block (A) was photographed for gross anatomical observation, whereas the lateral parasagittal block (B) was prepared for histological assessment in the manner detailed below. Firstly, the dorsal aspect of the sample block, including both the dermal and epidermal components, was removed by incision along the interface between the dorsal aspect of the distal phalanx and the laminar corium using a trimming knife. Specimen blocks for histological examination were prepared midway between the distal hairline in the coronary band and the bearing border of the hoof wall. This location was referred to as proximo-distal $50 \%$ hoof wall height (HWH) [28]. From this reference location, two transverse cuts were made. Cut one was made at 50\% HWH and cut two, $10 \mathrm{~mm}$ distal to $50 \% \mathrm{HWH}$. This produced a specimen block of 10 $\mathrm{mm}$ height that contained the Stratum medium (SM) of the hoof wall and the interdigitation of the epidermal and dermal lamellae. Finally the $S M$ was removed to produce a specimen block containing the LR. The specimen block was subsequently prepared for histological assessment by both light microscopy and transmission electron microscopy (TEM). For TEM examination, a sample from the medial aspect of block was trimmed to produce a tiny specimen $(2$ $\times 2 \times 1 \mathrm{~mm}$ ) of the deep area of the LR. This was immediately placed in buffered fixative.

The remaining portion of the specimen block was placed in Methacarn fixative prior to preparation for light microscopy and immunohistochemistry.

Histological and immunohistochemical staining: After paraffin sections $(6 \mu \mathrm{m})$ were prepared according to the usual procedures, hematoxylin and eosin (HE) staining, periodic acid-Schiff (PAS) reaction and immunohistological staining by the peroxidase anti-peroxidase (PAP) method were performed. Before immunohistochemical staining, sections were pretreated with $0.1 \%$ pepsin solution for unmasking antigen of the anti-type IV collagen polyclonal antibody [13], and with 6M-urea solution for unmasking both antigens of the anti-human type VII collagen monoclonal antibody (Biogenesis Ltd., Kingston, NH, U.S.A.) and the anti-human laminin 5 polyclonal antibody [15]. After reacting with the each primary antibody overnight at $4^{\circ} \mathrm{C}$, the sections were washed with Tris-buffered saline $(\mathrm{pH}$ 7.6) and then reacted with the secondary antibody, anti-rabbit or anti-murine IgG antibody (DAKO Japan, Kyoto, Japan) for $1 \mathrm{hr}$ at $37^{\circ} \mathrm{C}$. Finally, the sections were incubated with rabbit PAP or murine PAP (DAKO Japan, Kyoto, Japan) for $1 \mathrm{hr}$ at $37^{\circ} \mathrm{C}$, and colored with diaminobenzidine with hydrochloric acid and hydrogen peroxide.

Transmission electron microscopy: For TEM, the trimmed samples were pre-fixed using $2.5 \%$ glutaraldehyde plus $2 \%$ paraformaldehyde- cacodilate buffered solution (pH 7.4) for $2 \mathrm{hr}$ at $4^{\circ} \mathrm{C}$. After post-fixation in $1 \%$ osmium tetroxide and dehydration through a graded ethanol series, the samples were immersed in QY-1 (Oken, Tokyo, Japan). Then, tissues were embedded in a fresh mixture of Epok 812 , nadic methyl anhydride, dodecenyl succinic anhydride and DMP-30 (Oken, Tokyo, Japan). Ultrathin sections were double-stained with $2 \%$ uranyl acetate and $0.2 \%$ lead citrate and the grids were examined using a TEM (Hitachi H-7100, Tokyo, Japan) with at an acceleration voltage of $75 \mathrm{kV}$.

\section{RESULTS}

Gross anatomical assessments of parasagittal block (A) revealed DP rotation and widening of the LR with the lamellar wedge in all the laminitis cases, but not in the control hoof. These macroscopic observations were consistent with evidence of laminitis as previously published [18]. Nevertheless, the macroscopic fissure between the SM and the LR, which is often recognized in catastrophic laminitis [21], was not seen in any cases.

In the histological observation of control tissue, there was no abnormality in the LR. On the other hand, the regenerated LE was observed to have PEL with thickened layers of prickle cells and SELs in all laminitis cases (Fig. 1b-e). This thickened prickle cell layers (Fig. 1b) had a similar structure to that previously reported regarding the terminal papilla region in the normal hoof $[6,24]$. In Case 1 , the keratinized region of the PEL showed degenerative change including epithelial vacuolization adjacent to the apical tip of the LD (Fig. 1d). In all laminitis cases, microscopic gaps were observed inside of the $S M$ (Fig. 1a, e), although fissure formation between the $S M$ and LR was not seen macroscopi- 

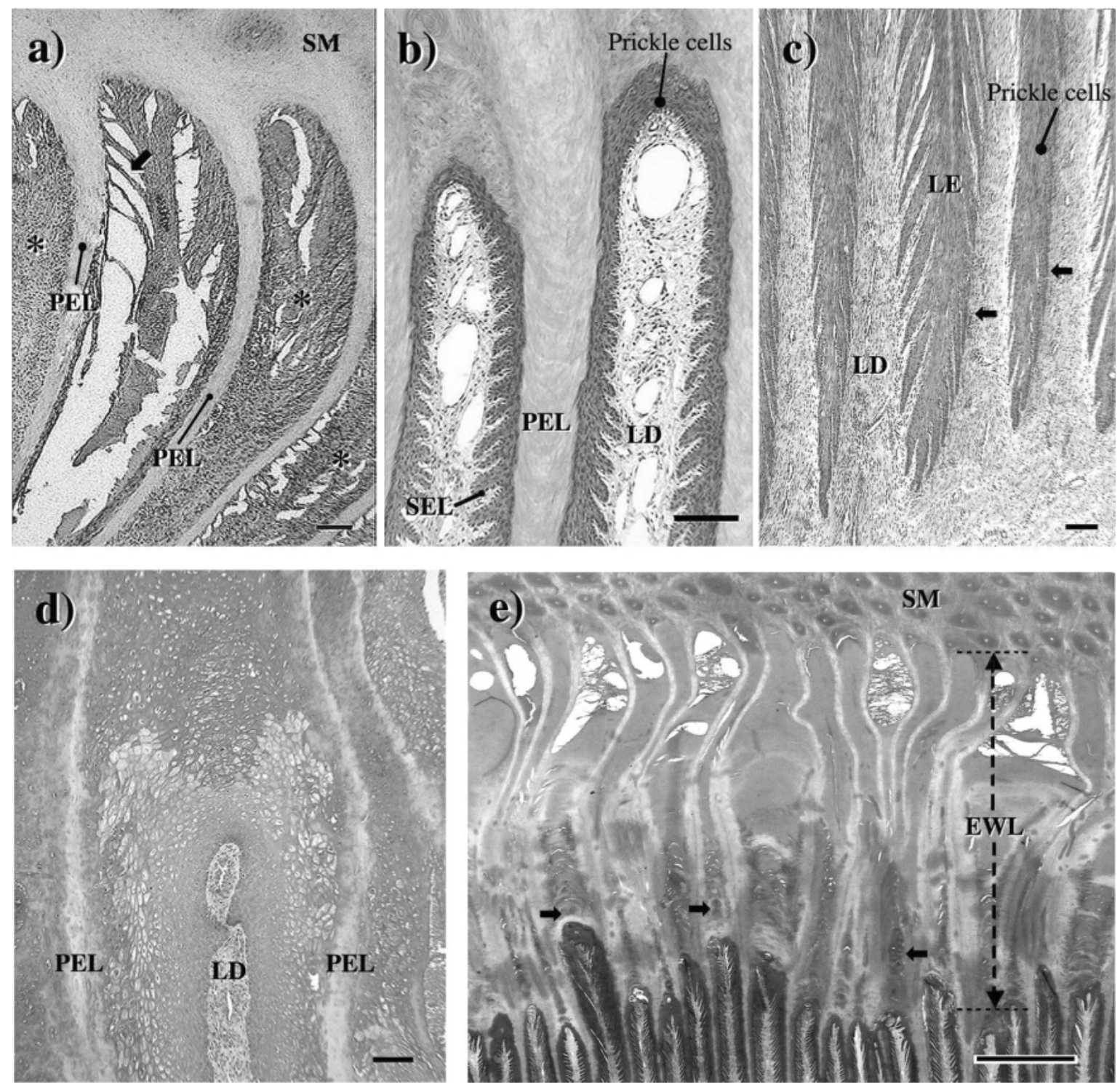

Fig. 1. Horizontal sections of the laminar region (HE stain). In these figures, its upper part indicates superficial area of the hoof and its lower part indicates deeper area nearby the distal phalanx. a) Case 1: It is observed that gaps are formed between the primary epidermal laminae inside the Stratum medium. In these submural gaps, there are filled with erythrocytes, keratinocyte debris, blood plasma (asterisks) and some degenerated secondary epidermal laminae (arrow). Bar=100 $\mu \mathrm{m}$. b) Case 2: The prickle cells make stratified layers surrounding the laminar dermis, which is similar structure with the terminal laminar region (the terminal papilla). Bar $=100 \mu \mathrm{m}$. c) Case 2: In the deep area of the laminar region, the secondary epidermal laminae are not well developed on the hemi-lateral side of the laminar epidermis (arrows). Bar=100 $\mu \mathrm{m}$. d) Case 1: Degenerative change including epithelial vacuolization is observed at the area alongside the tip of the laminar dermis. Bar=100 $\mu \mathrm{m}$. e) Case 3: There is observed the ectopic white line tissue having horn tubules (arrows) between the regenerated laminar epidermis and the Stratum medium. Bar: $1 \mathrm{~mm}$. SM; Stratum medium, PEL; primary epidermal laminar, SEL; secondary epidermal laminar, LE; laminar epidermis, LD; laminar dermis, EWL; ectopic white line.

cally. These submural gaps were oval to irregular in shape, and often contained the degenerated SELs attached to the PELs (Fig. 1a). The submural gaps contained erythrocytes, keratinocyte debris and blood plasma (Fig. 1a). In Cases 2 and 3, an ectopic white line (EWL) tissue having irregularly shaped horn tubules [18] was observed between the regener- ated LEs and those gaps (Fig. 1e). In the most inner region of the LR, the PELs were composed of non-keratinized epidermal tissue (Figs. 1c, 2a). In addition, the SELs were either not well developed (undeveloped SEL) or were absent on unilateral side of the most PELs (Figs. 1c, 2, 3). The SELs on the opposite side of those PELs were, however, rel- 

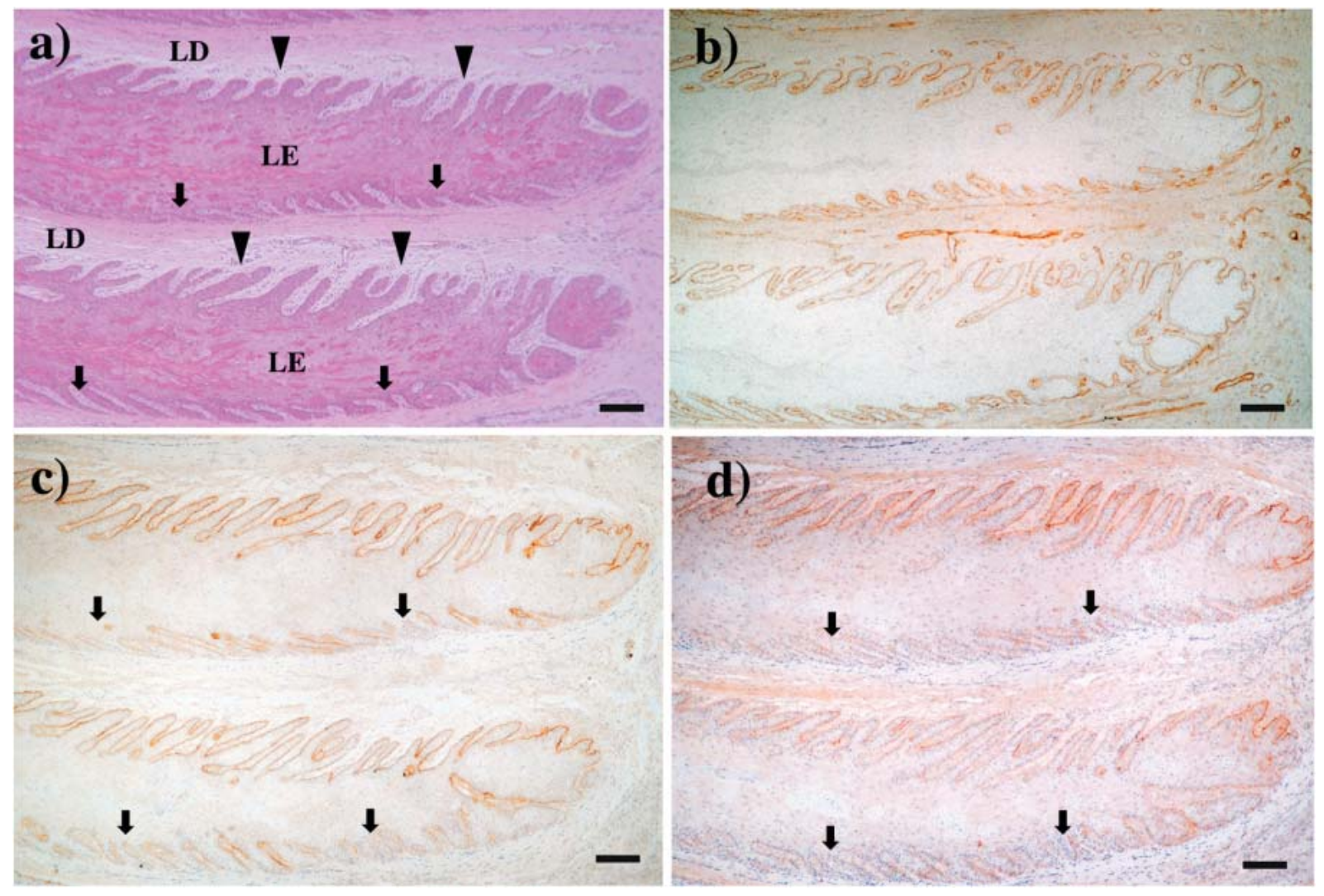

Fig. 2. Histological and immunohistochemical results in Case 1. In these figures, left indicates more superficial and right is deeper in the hoof. a) HE staining result. b) Immunohistochemical result for type IV collagen. c) Immunohistochemical result for type VII collagen. d) Immunohistochemical result for laminin 5. It is observed that the laminar epidermis is regenerated (a). Arrows and arrowheads in a) indicate in the undeveloped secondary epidermal laminae (ud-SELs) and well grown SELs (wg-SELs), respectively. Density of the dermis alongside the ud-SELs is higher than that alongside of wg-SELs. Immunohistochemical result for type IV collagen (b) on the same area of a) shows positive reaction in all edges of the secondary epidermal laminae. However, immunohistochemical results for type VII collagen and laminin 5 show negative reaction only in the BMZ alongside the ud-SELs indicated by arrows in c) and d). Bar $=100 \mu \mathrm{m}$. LD; laminar dermis, LE; laminar epidermis.

atively well grown. There was no exfoliative change evidence between these undeveloped SEL (ud-SEL) and the dermis. In the ud-SEL of Cases 1 and 2, the density of collagen fibers in the dermis adjoining the ud-SEL was high (Fig. 2a). PAS reaction showed the presence of the basement membrane in most border regions between the LE and the LD in all cases. In Cases 2 and 3, there were much inflammatory mononuclear cells in the LD (Fig. 1c), but Case 1 showed that a few inflammatory cells had invaded in the dermis.

In immunohistochemical observations of the control tissue, all BMZs in the laminar interface were positive for type IV collagen, type VII collagen and laminin 5. In laminitis cases, BMZ staining results for type IV collagen were positive (Fig. 2b) as well as the control one. In addition, the BMZ stained positive for both anti-type VII collagen antibodies and anti-laminin 5 antibodies, except for the BMZ adjoining ud-SEL (Figs. 2c, d, 3a, b). In some areas in Cases 2 and 3, type VII collagen positive and laminin 5 negative BMZs were observed. Only in Case 3, there were parts of the well grown SELs (wg-SELs) showing negative BMZ for both of type VII collagen and laminin 5 (Fig. 3c, d). These location corresponded with the location of the udSELs on the other PELs.

Transmission electron microscopy of the ud-SEL showed the lamina densa and the lamina lucida were present in all cases at the boundary between the dermis and epidermis. However, the anchoring fibrils and the hemidesmosomes were either absent, or present at lower than normal levels in those regions (Fig. 4). In contrast, the most BMZ of wgSELs showed same ultrastructures as the normal BMZ of control case. Only in Case 3, some parts of the BMZ in wgSEL showed same abnormal ultrastructures as the BMZ in ud-SEL. It might coincide with the aforementioned immunohistochemically negative BMZ against type VII collagen and laminin 5 in the wg-SEL in Case 3.

\section{DISCUSSION}

Histological evidence of the submural gaps containing 

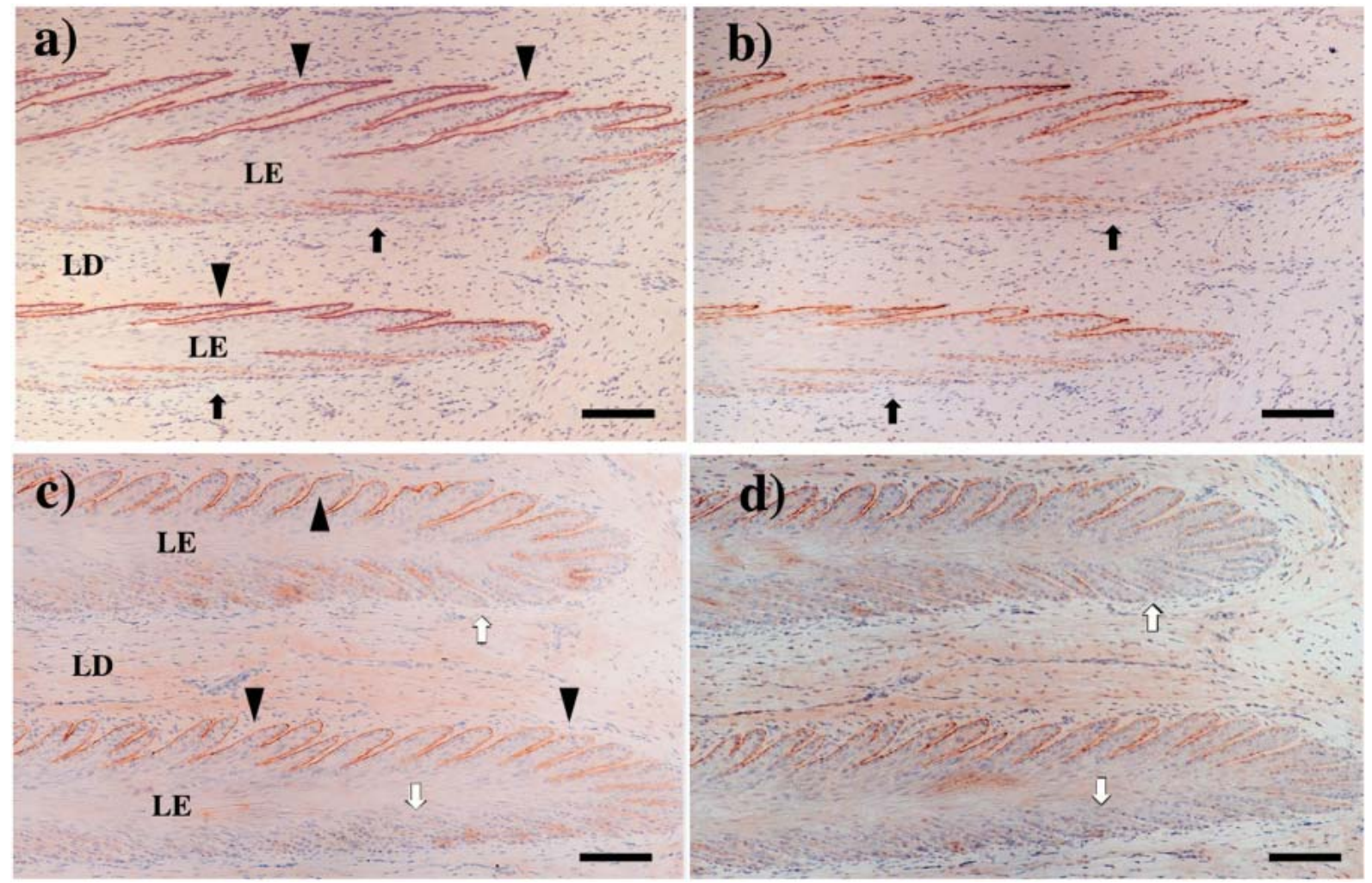

Fig. 3. Immunohistochemical results in Cases 2 and 3. In these figures, left indicates more superficial and right is deeper. a) Immunohistochemical result for type VII collagen in Case 2. b) Immunohistochemical result for laminin 5 in Case 2. c) Immunohistochemical result for type VII collagen in Case 3. d) Immunohistochemical result for laminin 5 in Case 3. Negative reaction for type VII collagen and laminin 5 are seen in unilateral side of the laminar epidermis, which corresponds to the undeveloped secondary epidermal laminae in Case 2 (arrows). The arrowheads indicate in well grown SELs (wg-SELs) in the both cases. Only in some wg-SELs in Case 3 (white arrows), negative reaction can be seen. Bar $=100 \mu \mathrm{m}$. LD; laminar dermis, LE; laminar epidermis.

the remnant of degenerative SELs suggested that detachment of the LE from the LD had occurred at the laminar interface during the initial stages of laminitis $[18,26]$. Anatomically, one to two prickle cell layers is observed in the epidermal lamina of the normal hoof [24]. However, the regenerated LE of the chronically affected hoof had an stratified prickle cell layers. This morphological characteristic of the studied cases was similar to the terminal papilla region of the normal hoof, which can produce the white line tissue [6]. This fact suggests that the healing process in laminitis is associated both with a progressive proliferation of keratinocytes, and also a metaplastic response from the original structure of LE to the terminal papilla like tissue. This metaplastic response of the LE may lead to generation of the ectopic white line tissue in the space between the regenerated LR and the $S M$ at $50 \% \mathrm{HWH}[6,18]$. The parts of SEL were not well developed in some area, especially in the unilateral side of PEL in the inner region of LR. Though its cause could not be identified in this study, possible explanations could be as follows. It is well known that epidermal growth in human cell culture is influenced and enhanced when the collagen matrix had previously been reorganized by fibroblasts, and is greatest when intact living fibroblasts existed in this matrix [8]. In other words, if abnormal fibroblast or degenerative collagen matrix existed under the keratinocyte, the keratinocyte proliferation was abnormally influenced. The high collagen- density area of the LD observed in this study, which might relate with degeneration of fibroblasts and abnormal remodeling of collagen fibers, could mislead epidermalization through false dermo-epidermal interaction. As the result, some misled keratinocytes displayed functional abnormality in cell division resulting in constructing dwarfish of the SEL (i.e., ud-SEL). Another explanation may be that DP rotation stretched the LE, resulting in mechanical stress. This force might compress the dermis and vessels, so that adequate nutrition and oxygen could not be supplied to the part of keratinocytes, resulting in the generation of ud-SEL. Compressed dermis by mechanical stress also could show partial high collagen-density area in the LD.

Type IV collagen, type XII collagen and laminin 5 are important structural protein components of the BMZ [2, 4, 11]. Extracellular accumulation of the type IV collagen results in membrane formation, through self-assembly of a mesh-like sheet, which is ultramicroscopically seen as the lamina densa in the $\mathrm{BMZ}[1]$. Other components are 


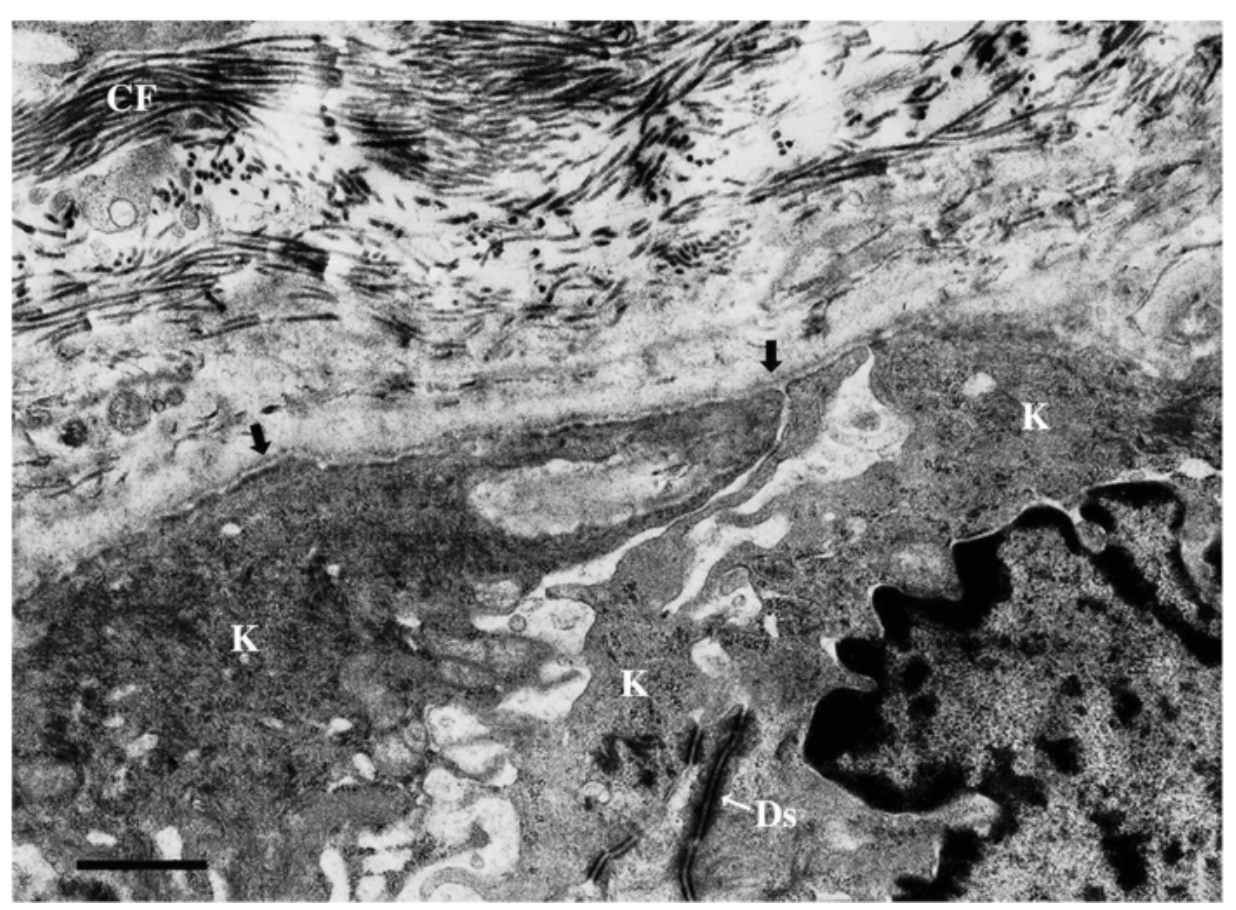

Fig. 4. Transmission electron microscopic observations of the basement membrane zone adjoining the keratinocytes composing undeveloped secondary epidermal laminar in Case 1. There are observed the lamina densa (arrows) and the lamina lucida, but no anchoring fibrils and few hemidesmosomes in the basement membrane zone. Bar: $900 \mathrm{~nm}$. Ds; desmosome, CF; collagen fibril, K; keratinocyte.

attached to this mesh-like sheet and completed to assemble the BMZ [2]. The hemidesmosome and the anchoring fibril are also important ultrastructures within the BMZ, which serve to unite the epidermis and dermis [4, 23]. Laminin 5 and type XVII collagen are thought to constitute the hemidesmosome $[3,20]$, and type VII collagen is a major component of anchoring fibrils [32]. With immunohistochemical analysis of the present study, the presence of the basement membrane was verified in most regions by type IV collagenpositive reactions. This agreed well with the results of the PAS reaction and evidence of the ultramicroscopic BM existence. However, type VII collagen and laminin 5 indicated negative in some parts of the BMZ, particularly alongside the ud-SELs. These observations were in agreement with the ultramicroscopic results, which showed a smaller number and/or absence of the hemidesmosomes and the anchoring fibrils in those areas. These phenomena might indicate that the keratinocytes comprising the regenerated LE has functional abnormality. Alternatively, activation of the reported MMPs relating with laminitis onset could dissolve the hemidesmosomes and anchoring fibrils in the BMZ even if it was promoting in the chronic stage [14]. However, latter explanation contradicts the evidence in which the intact lamina densa was seen in the BMZ through the TEM, because the reported MMPs can destroy not only type VII collagen or laminin 5 but also type IV collagen [14, 27]. Further work is required for aspects of this immunohistochemical abnormality and the MMP activation. By the way, only in Case 3, the BMZ in parts of wg-SEL showed negative for both of type VII collagen and laminin 5. It can be considered that some parts of ud-SEL had grown and formed to the wg-SEL in comparatively long healing process, but generation of the type VII collagen and the laminin 5 were still not occurred in that area.

Furthermore, the light microscopic observation did not show exfoliative change within the abnormal BMZ although type VII collagen and laminin 5 were present only in very small amounts, or were indeed absent. This observation might suggest that binding elements other than the proteins examined in this study could be involved in the dermo-epidermal junction of the equine LR. As a similar thing, it is known that the intestine has no anchoring fibrils and little laminin 5. Therefore, it is considered that unknown proteins may bind the mucoepithelium and the lamina propria [30].

In conclusion, the present study indicated that the regenerated BMZ of the chronic laminitic foot differed from one observed within the normal foot. In the unilateral side of ud-SELs of the regenerated LR in chronic laminitis, BMZ structure was not fully constructed. This indicates that the regenerated LR might not have the ability to form BMZ structures and/or that type VII collagen and laminin 5 might have electively dissolved only in those ud-SELs in some mechanism. That fact might be related with recurrence nature of this disease. However this study reports the findings of only three cases. Further studies are required to establish whether the findings relate to all laminitis cases. 
ACKNOWLEDGMENTS. We thank Dr. Burgeson, R. E. (Cutaneous Biology Research Center, Massachuusetts General Hospital, and the Department of Dermatology, Harvard Medical School) for providing polyclonal antibodies against laminin 5. Further, we thank Dr. Reilly, J. D. and Dr. Collins, S. N. (Division of Nutritional Biochemistry, School of Biosciences, the Nottingham University) for proofreading this article to appropriate English.

\section{REFERENCES}

1. Adachi, E., Takeda, Y., Nakazato, K., Muraoka, M., Iwata, M., Sasaki, T., Imamura, Y., Hopkinson, I. and Hayashi, T. 1997. Isolated collagen IV retains the potential to form an 18-nm sided polygonal meshwork of the lamina densa. J. Electron Microsc. 46: 233-241.

2. Adachi, E., Hopkinson, I. and Hayashi, T. 1997. Basementmembrane stromal relationships: interactions between collagen fibrils and the lamina densa. Int. Rev. Cytol. 173: 73-156.

3. Aho, S. and Uitto, J. 1999. 180-kD bullous pemphigoid antigen/type XVII collagen: tissue-specific expression and molecular interactions with keratin 18. J. Cell Biochem. 72: 356-367.

4. Alberts, B., Bray, D., Lewis, J., Raff, M., Roberts, K. and Watson, D. J. 1994. Cell junctions, cell adhesion and the extracellular matrix. pp. 950-1009. In: Molecular Biology of the Cell (Alberts, B. ed.), Garland Publishing, Inc., New York.

5. Baxter, M. G. and Schlipf, W. J. 1995. Handling, restraint, and clinical evaluation. pp. 23-34. In: The Horse: Disease and Clinical Management (Kobluk, N. C., Ames, R. T. and Geor, J. R. eds.), W.B. Saunders Company, Philadelphia.

6. Budras, K. D., Hullinger, R. L. and Sack, W. O. 1989. Light and electron microscopy of keratinization in the laminar epidermis of the equine hoof with reference to laminitis. Am. J. Vet. Res. 50: 1150-1160.

7. Chetty, B. V., Boissy, R. E., Warden, G. D. and Nordlund, J. J. 1992. Basement membrane and fibroblast aberration in blisters at the donor, graft, and spontaneously healed sites in patients with burns. Arch. Dermatol. 128: 181-186.

8. Coulomb, B., Lebreton, C. and Dubertret, L. 1989. Influence of human dermal fibroblasts on epidermalization. J. Invest. Dermatol. 92: 122-125.

9. Cripps, J. P. and Eustace, A. R. 1999. Factors involved in the prognosis of equine laminitis in the UK. Equine Vet. J. 31: 433-442.

10. Epstein, A., Hendrick, S. J., Sanchez, R. L., Solomon, A. R. and Fine, J. D. 1988. Persistent subepidermal blistering in splitthickness skin graft sites. Ultrastructural and antigenic features simulating dystrophic or immunofluorescence-negative acquired epidermolysis bullosa. Arch. Dermatol. 124: 244249.

11. Fleischmajer, R., Utani, A., MacDonald, E. D., Perlish, J. S., Pan, T. C., Chu, M. L., Nomizu, M., Ninomiya, Y. and Yamada, Y. 1998. Initiation of skin basement membrane formation at the epidermo-dermal interface involves assembly of laminins through binding to cell membrane receptors. J. Cell. Sci. 111: 1929-1940.

12. Hood, M. D. 1999. Laminitis in the horse. Vet. Clinics of North Am. 15: 287-294.

13. Itoh, M., Arai, K. and Uehara, K. 1994. Localization of type IV collagen in the myotome cells during the somite differentiation in the chick embryo. Dev. Growth Differ. 36: 427-435.
14. Johnson, P. J., Tyagi, S. C., Katwa, L. C., Ganjam, V. K., Moore, L. A., Kreeger, J. M. and Messer, N. T. 1998. Activation of extracellular matrix metalloproteinases in equine laminitis. Vet. Rec. 11: 392-396.

15. Kadoya, Y. and Yamashina, S. 1999. Localization of laminin 5, HD1/plectin, and BP230 in the submandibular glands of developing and adult mice. Histochem. Cell Biol. 112: 417-425.

16. Kameya, T., Kiryu, K. and Kaneko, M. 1980. Histopathogenesis of thickening of the hoof wall laminae in equine laminitis. Jpn. J. Vet. Sci. 42: 361-371.

17. Konig, A., Lauharanta, J. and Bruckner-Tuderman, L. 1992. Keratinocytes and fibroblasts from a patient with mutilating dystrophic epidermolysis bullosa synthesize drastically reduced amounts of collagen VII: lack of effect of transforming growth factor-beta. J. Invest. Dermatol. 99: 808-812.

18. Kuwano, A., Katayama, Y., Kasashima, Y., Okada, K. and Reilly, D. J. 2002. A gross and histopathological study of an ectopic white line development in equine laminitis. J. Vet. Med. Sci. 64: 893-900.

19. Macfarlane, A.W. and Verbov, J. L. 1989. Trauma-induced bullous pemphigoid. Clin. Exp. Dermatol. 14: 245-249.

20. McGrath, J. A., Gatalica, B., Christiano, A. M., Li, K., Owaribe, K., McMillan, J. R., Eady, R. A. and Uitto, J. 1995. Mutations in the $180-\mathrm{kD}$ bullous pemphigoid antigen (BPAG2), a hemidesmosomal transmembrane collagen (COL17A1), in generalized atrophic benign epidermolysis bullosa. Nat. Genet. 11: 83-86.

21. Morgan, J. S., Grosenbaugh, A. D. and Hood, M. D. 1999. The pathophysiology of chronic laminitis. Vet. Clinics of North Am. 15: 395-417.

22. Mungall, A. B., Pollitt, C. C. and Collins, R. 1998. Localization of gelatinase activity in epidermal hoof lamellae by in situ zymography. Histochem. Cell Biol. 110: 535-540.

23. Pollitt, C. C. 1994. The basement membrane at the equine hoof dermal epidermal junction. Equine Vet. J. 26: 399-407.

24. Pollitt, C. C. 1995. Structure and function. pp. 7-45. In: Color Atlas of the Horse's Foot (Pollitt, C. C. ed.), Mosby-Wolfe, London.

25. Pollitt, C. C. 1995. Laminitis. pp. 175-177. In: Color Atlas of the Horse's Foot (Pollitt, C. C. ed.), Mosby-Wolfe, London.

26. Pollitt, C. C. 1996. Basement membrane pathology : a feature of acute equine laminitis. Equine Vet. J. 28: 38-46.

27. Pollitt, C. C. and Daradka, M. 1998. Equine laminitis basement membrane pathology: loss of type IV collagen, type VII collagen and laminin immunostaining. Equine Vet. J. (Suppl.) 26: 139-144.

28. Reilly, J. D., Cottrell, D. F., Martin, R.J. and Cuddeford, D. 1996. Tuble density in equine hoof horn. Biomimetics 4: 23 36.

29. Roberts, D. E., Ochoa, R. and Haynes, F. P. 1980. Correlation of laminar dermal-epidermal laminar lesions of equine hoof with various disease conditions. Vet. Pathol. 17: 656-666.

30. Simon-Assmann, P., Kedinger, M., De Arcangelis, A., Rousseau, V. and Simo, P. 1995. Extracellular matrix components in intestinal development. Experientia 51: 883-900.

31. Su, J. C., Varigos, G. A. and Dowling, J. 1998. Epidermolysis bullosa acquisita in childhood. Australas. J. Dermatol. 39: 3841.

32. Woodley, D. T., Burgeson, R. E., Lunstrum, G., BrucknerTuderman, L., Reese, M. J. and Briggaman, R. A. 1988. Epidermolysis bullosa acquisita antigen is the globular carboxyl terminus of type VII procollagen. J. Clin. Invest. 81: 683-687. 